\title{
The primary energy spectrum derived from Linsley method with simulations of heavy compositions in the LAAS mini array observation
}

Hiroki Matsumoto ${ }^{* a}$, Atsushi lyono $^{b}$, Kazuhide Okei $^{a}$, Shuhei Tsuji ${ }^{a}$, Soji Ohara ${ }^{c}$, Nobuaki Ochi ${ }^{d}$, Nobusuke Takahashi ${ }^{e}$, Isao Yamamoto ${ }^{f}$, Takao Nakatsuka ${ }^{g}$ and Saya Yamamoto ${ }^{h}$,

${ }^{a}$ Kawasaki Medical School

Kurashiki 701-0192, Japan

${ }^{b}$ Dept. of Fundamental Science, Faculty of Science, Okayama university of Science

Okayama 700-0005, Japan

${ }^{c}$ Nara Sangyo University

Nara 636-8503, Japan

${ }^{d}$ Toyo University

Tokyo 112-8606, Japan

${ }^{e}$ Dept. of Physics, Hirosaki University

Hirosaki 036-8560, Japan

${ }^{f}$ Dept. of Information and Computer Engineering, Okayama University of Science

Okayama 700-0005, Japan

${ }^{g}$ Okayama Shoka University

Okayama 700-8601, Japan

${ }^{h}$ Dept. of Fundamental Science, Okayama University of Science

Okayama 700-0005, Japan

E-mail: h.matsumoto@med.kawasaki-m.ac.jp iyonoddas.ous.ac.jp

The primary energy and its spectrum are obtained by using compact Extensive Air Shower (EAS) arrays, the apparatus for restricting the EAS zenith angle, and the Linsley method. In this work, we carried out the simulation and the data analysis by taking into account of proton and iron primaries. The primary spectral indices $\alpha$ (proton) and $\alpha$ (iron) are obtained as $\alpha$ (proton) $=-2.47 \pm 0.17$ and $\alpha$ (iron) $=-3.38 \pm 0.25$, respectively, in the single observation of a compact EAS array, in the primary energy region of $10^{16} \mathrm{eV}$ to $10^{18.5} \mathrm{eV}$. Additionally, $\alpha$ (proton) $=-3.12 \pm 0.13$ and $\alpha$ (iron) $=-3.52 \pm 0.17$ are obtained with each composition in the synchronized observation between the compact EAS array and the apparatus for restricting the EAS zenith angle in the same primary energy region.

The 34th International Cosmic Ray Conference,

30 July- 6 August, 2015

The Hague, The Netherlands

\footnotetext{
* Speaker.
} 


\section{Introduction}

Investigations for high energy cosmic rays have been performed by various observations such as Auger [1], Telescope Array [2], Yakutsk [3] and so on, in order to understand questions for the highest cosmic ray energy, the composition of cosmic rays, the spectral index, changes of that etc. In Okayama University of Science, we have been studied and observed cosmic rays since 1990s. Especially, the searching for long-range coincidence events among Extensive Air Shower (EAS) arrays installed in several sites has been performed [4, 5, 6.

Generally, the estimation for the primary energy of EASs is required with the core distance $r$ and local particle density $\rho$ in an observation method of arranging scintillation counters on the ground, therefore many detectors and a larger area are required. Our arrays are designed as the mini array constructed of 8 scintillation counters and covered a surface of about $200 \mathrm{~m}^{2}$. Thus, it is difficult to obtain the core distance $r$ for our arrays, and the primary energy estimation is hard by using classical methods. To resolve this problem, we focused the dependence of the core distance $r$ on the dispersion $\sigma_{\mathrm{t}}$ of arrival time of EAS particles, and were able to obtain the core distance event by event. The relation between $r$ and $\sigma_{\mathrm{t}}$ was reported by Linsley et. al [7]. We have observed the EAS arrival time with a mini array since 2006 [8], and set the apparatus for restricting EAS zenith angle in order to improve the determination accuracy of the primary energy in 2010 [9].

We report simulation results and the obtained data spectrum in the primary energy region of $10^{16} \mathrm{eV}$ to $10^{19.5} \mathrm{eV}$ assumed proton and iron primaries.

\section{Apparatus}

The observation of cosmic rays in Okayama University of Science (OUS) has five EAS arrays, OUS1, OUS2, OUS3, OUS4 and OUS5, in Okayama city, Japan [6. These are located at $34^{\circ} 42^{\prime}(\mathrm{N})$ and $133^{\circ} 56^{\prime}(\mathrm{E})$, at sea level. OUS arrays except the OUS4 typically consist of 8 scintillation counters. Each counter has a scintillator of which size is $50 \mathrm{~cm} \times 50 \mathrm{~cm} \times 5 \mathrm{~cm}$, and a PMT, and is sealed in a stainless steel case ( $5 \mathrm{~mm}$ thickness). These arrays are installed on the rooftop of the building in the university, and OUS5 is set on a botanical garden located at distance of about $2 \mathrm{~km}$ from the university. OUS arrays except the OUS4 are typical surface detectors. The OUS4 is used to obtain zenith angle information of EAS and is installed in the first floor of a four stories building. In this work, we use data of the OUS1 and the OUS4.

The OUS1 array covers a surface of about $200 \mathrm{~m}^{2}$ and its location is shown in Figure 1. (a). The data acquisition system (DAQ) provides a TDC, a ADC, and a shift register [9]. A GPS moduleis used for a time stamp of event trigger and clock synchronization. The time accuracy is $1 \mu \mathrm{s}$ because of $10 \mathrm{MHz}$ oscillator maintained by receiving 1 pps GPS signal. An event trigger signal is generated by the coincidence module when more than three detectors are hit within 2.5 $\mu \mathrm{s}$. The shift register module is used for recording timing signal from each detector as a digital timing diagram. The time window is set to $\pm 2.5 \mu$ s from an event trigger time. The time resolution of this shift register module is equal to $5 \mathrm{~ns}$. The arrival time of each EAS particle is determined by combining an event trigger time given by the GPS with a digital timing diagram in the shift register. We use the shift register module instead of the TDC module, for the purpose of observing the arrival time distribution from a shower front to a shower tail. Originally, the OUS1 is designed 
to reconstruct an arrival direction of EAS by using the TDC. However, since the uncertainty of estimation of EAS arrival direction increases with the dispersion of arrival time of EAS, the arrival direction cannot be determined by using the TDC. And especially, we focus on events associated with the large dispersion of arrival time of EAS particles. Thus we restrict the EAS zenith angle range by using the OUS4 detectors.

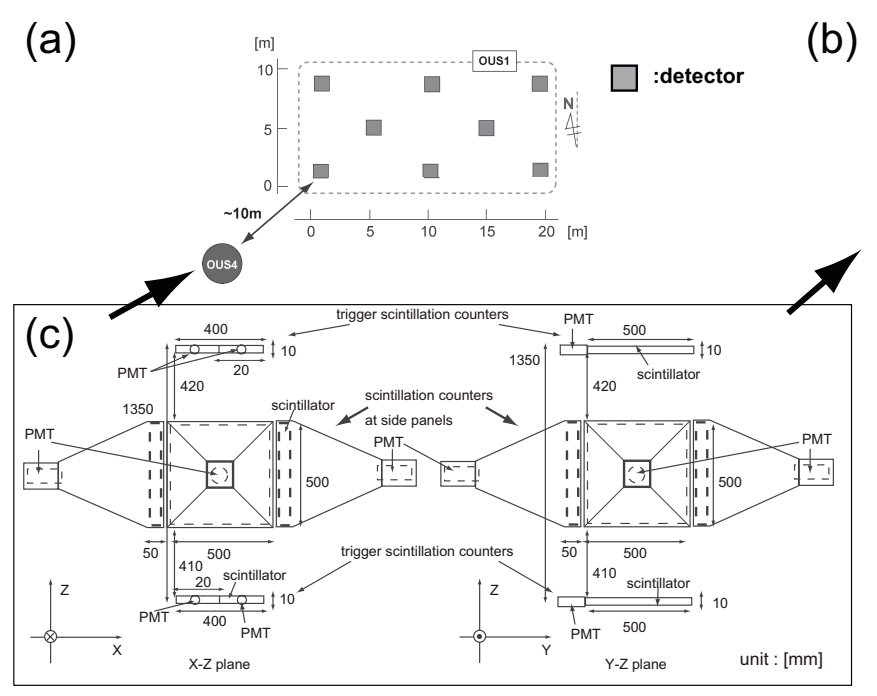

(b)

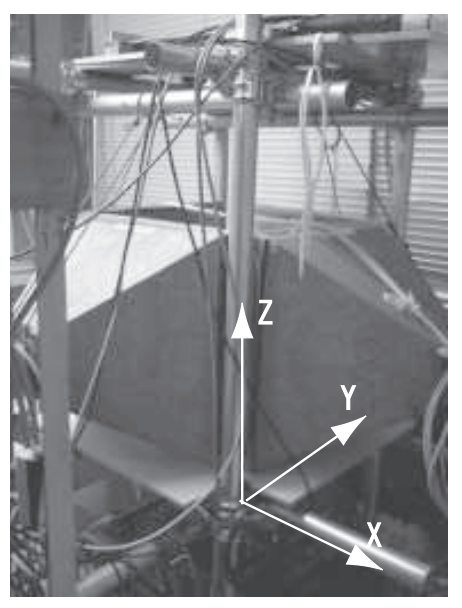

Figure 1: (a) The layout of the OUS1 array and the OUS4 array. (b) The photograph of the OUS4. (c) The schematic drawing of the OUS4.

Figure 1 (b) and1 1 (c) show the photograph and the schematic drawing of OUS4 array, respectively. The OUS4 is equipped with two scintillation counters, of which size is $20 \mathrm{~cm} \times 50 \mathrm{~cm} \times$ $1 \mathrm{~cm}$, with a PMT and a black plastic cover, at the top layer and the bottom layer, respectively. An EAS trigger signal is generated with a coincidence between the scintillation counters at the top layer and at the bottom layer. EAS event data are collected with the same DAQ of the OUS1. The observation time is synchronized by the GPS system. The scintillation counters at side panels are the same as those of the OUS1 and are installed to restrict the EAS zenith angle range. The counters are enabled to distinguish the EAS events with the zenith angle $25.6^{\circ}$ among all events.

\section{Estimation of the core distance}

In order to obtain the core distance $r$ and the local particle density $\rho$ with the OUS1, we apply the Linsley method to our compact array observation. The dispersion of arrival time distribution of each EAS particles is defined by $\sigma_{\mathrm{t}}$, and the average dispersion of observed events can be defined by $\left\langle\sigma_{\mathrm{t}}\right\rangle$. Linsley described $\left\langle\sigma_{\mathrm{t}}\right\rangle$ in following the empirical formula [7]

$$
\left\langle\sigma_{t}\right\rangle=\sigma_{t 0}\left(1+\frac{r}{r_{t}}\right)^{b}
$$

where $\sigma_{t 0}=1.6 \mathrm{~ns}, r_{t}=30 \mathrm{~m}, b=(2.08 \pm 0.08)-(0.4 \pm 0.06) \sec \theta+(0 \pm 0.06) \log \left(E / 10^{17} \mathrm{eV}\right)$, $\theta$ is the zenith angle and $E$ is the primary cosmic ray energy. We adapt $b$ to $\bar{b}=1.65$ averaged by 
the EAS zenith angle distribution expected by this observation, and ignore the contribution of the primary energy.

Linsley assumed that the EAS arrival time distribution is the gamma distribution. Then, $\sigma_{\mathrm{t}}$ of the distribution is obtained by

$$
\sigma_{\mathrm{t}}=\frac{\langle t\rangle^{2}}{2}
$$

where $t$ is the arrival time of EAS particle. However, we used the median $t_{\mathrm{m}}$ of observed data samples of EAS arrival time as the dispersion estimator instead of Eq. 3.2 [8]. Because Eq. 3.2 is overestimated by the contamination of random noise in the tail of EAS arrival time distribution [9]. Thus we define $\sigma_{\mathrm{t}}$ as

$$
\sigma_{\mathrm{t}}=\frac{\sqrt{2}}{1.67} t_{\mathrm{m}}
$$

After substitution of Eq. 3.3 into Eq. 3.1 and rearrangement of the terms, we obtain

$$
r=30\left(\left(1.35 t_{\mathrm{m}}\right)^{\frac{1}{1.65}}\right) .
$$

The relation between $r$ and $\left\langle\sigma_{\mathrm{t}}\right\rangle$ is shown in Figure $2\left\langle\sigma_{\mathrm{t}}\right\rangle$ mainly depends on $r$ and $\theta$. We use the averaged $b$ in this work. Note that one of systematic errors in the estimation $E$ arises from averaging the EAS zenith angle.

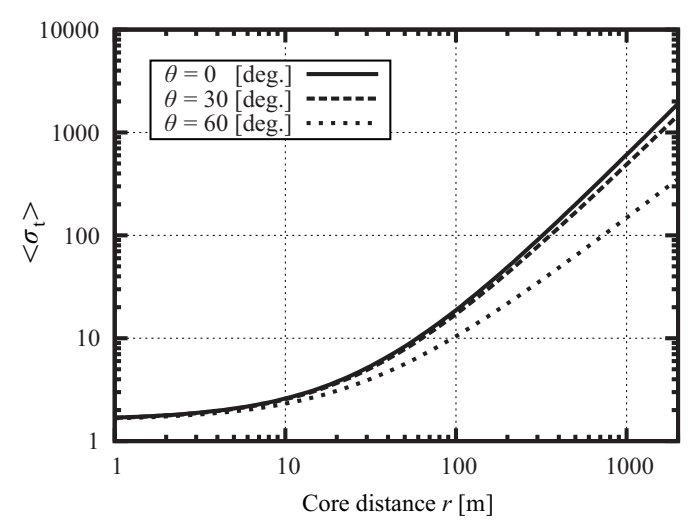

Figure 2: The relation between $r$ and $\sigma_{\mathrm{t}}$. EAS zenith angles are assumed to be $\theta=0^{\circ}, 30^{\circ}$ and $60^{\circ}$.

\section{Data analysis}

We use data obtained from 4/2006 to12/2012 in the single observation of the OUS1, and from $8 / 2008$ to $12 / 2012$ in the synchronized observation between the OUS1 and the OUS4 (OUS1+4). Data summary is listed in Table 1 Common criteria for this data analysis are the observed core distance $r_{\mathrm{obs}}>100 \mathrm{~m}$ and $r_{\mathrm{obs}}<2000 \mathrm{~m}$. In the OUS1+4, we set the coincidence criterion that the time difference between the OUS1 and the OUS4 is within $10 \mu$ s to identify the same EAS.

The primary energy is calculated from the observed local particle density $\rho_{\mathrm{obs}}, r_{\mathrm{obs}}$ and the simulated lateral distribution function. $\rho_{\mathrm{obs}}$ is obtained by $n / 2\left[/ \mathrm{m}^{2}\right]$, where $n$ indicates the number of particles obtained with the OUS1. $r_{\mathrm{obs}}$ is calculated by using Eq. 3.3 and the median of arrival time distribution calculated from data obtained with the shift register system. 


\begin{tabular}{|l|c|c|c|c|}
\hline & $T_{\text {obs }}$ [day] & $N_{\text {obs }}$ & $N_{\text {est1 }}$ & $N_{\text {est2 }}$ \\
\hline OUS1 & 1931 & $1.3 \times 10^{7}$ & 76571 & 161135 \\
\hline OUS1+4 & 1336 & $2.0 \times 10^{5}$ & 553 & 1876 \\
\hline
\end{tabular}

Table 1: The data summary of the OUS1 and the OUS4 observations. $T_{\mathrm{obs}}$ is total observation time. $N_{\mathrm{obs}}$ indicates the total number of triggered events. $N_{\text {est1 }}$ and $N_{\text {est2 }}$ indicate the total number of events estimated their primary energy $\left(E_{0} \geq 10^{16} \mathrm{eV}\right)$ assumed nuclei to be protons and irons, respectively.

\section{Result}

We carried out simulations and the data analysis taking account of iron primaries in this paper, because only proton primaries had been considered in previous studies. To perform detector simulations and the data analysis, the database of the shower size and the lateral distribution of electrons and muons are made by using the EAS simulation program AIRES [10]. QGSJET II-3 [11] and Hillas Spritting model [12] are used to hadronic interaction models. Input parameters of the EAS simulation are the primary energy $E_{0}$ and the zenith angle $\theta . E_{0}$ is sampled every one order of magnitude in the primary energy range of $10^{16} \mathrm{eV}$ to $10^{20} \mathrm{eV}$. $\theta$ is sampled every $10^{\circ}$ from $0^{\circ}$ to $60^{\circ}$. The database simulation and the detector simulation are described in detail in the reference [9]. Figure 3 shows the comparison of the proton and the iron results in the simulated energy distribution at $E_{0}=10^{17} \mathrm{eV}$. The energy distributions in iron primaries are shifted to the higher energy region than those in protons, because the lateral distribution function in the iron primary is lower than that in proton primaries in the same primary energy. Thus, the primary energy assuming iron primaries have been estimated to be higher than proton primaries when we determined values of $\rho_{\mathrm{obs}}$ and $r_{\mathrm{obs}}$ and the primary energy by using the lateral distribution.
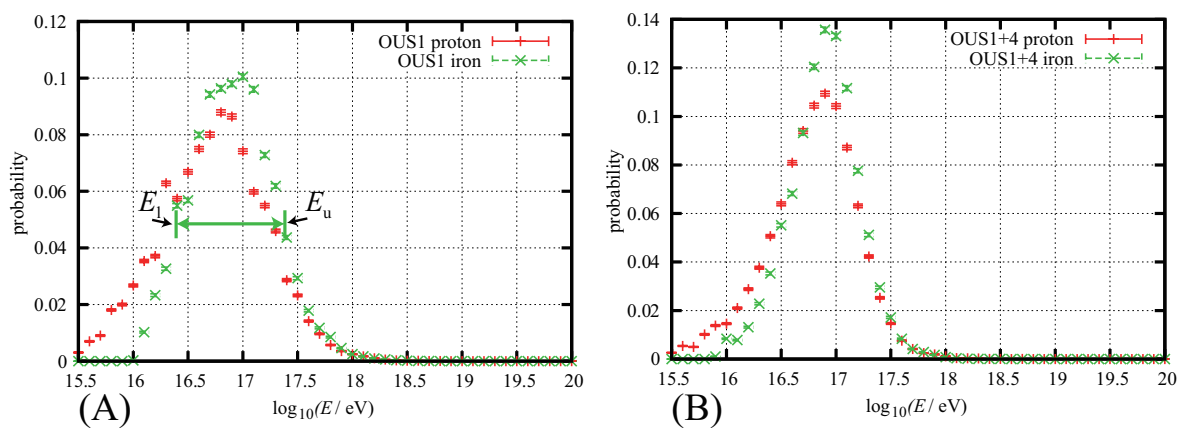

Figure 3: The comparison of proton primaries with irons in the simulated energy distribution in the OUS1 (A) and the OUS1+4 (B). The input primary energy is $E_{0}=10^{17} \mathrm{eV}$ with each simulation. Output energy $E$ is represented on the $\mathrm{x}$-axis.

We define the energy resolutions of the OUS1 and the OUS1+4 as the FWHM values of the energy distribution obtained by the detector simulation. $E_{1}$ and $E_{\mathrm{u}}$ in Figure 3 indicate the energy values at half maximum of the energy distribution. In Figure 4 we show the comparison of the energy resolution of proton primaries with that of irons. Below $E_{0}=10^{17.5} \mathrm{eV}$, each energy resolution differs due to lower particle density of lateral distribution in iron primaries. 

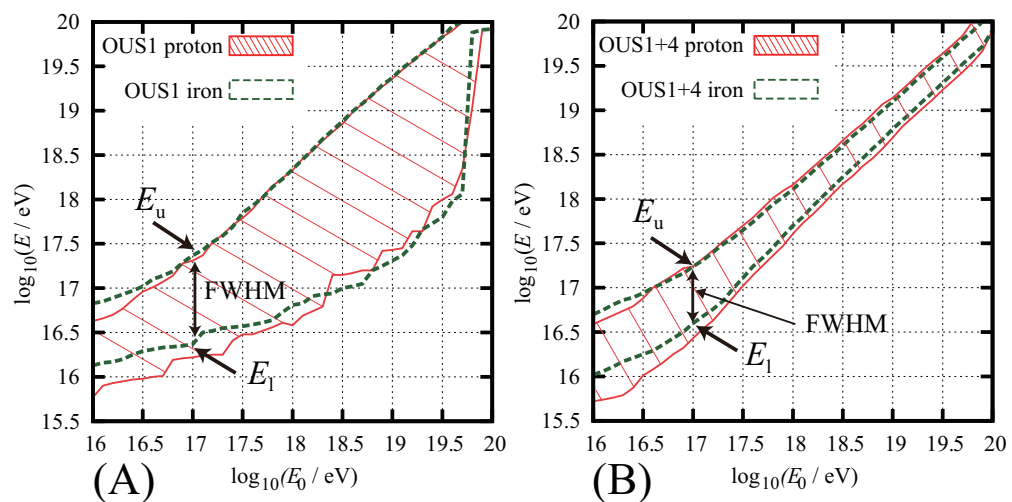

Figure 4: The comparison of proton primaries with irons in the energy resolution as a function of $E$ in the OUS1 (A) and the OUS1+4 (B). The shaded regions indicate the results of proton primaries. The regions surrounded by a dashed line indicate that of irons.

The primary energy spectrum in the simulation is assumed to be a single power law $E^{\alpha}$, where $\alpha$ is the spectral index. Actually, $\alpha$ is obtained as the observed value $\alpha^{\prime}$ due to the sensitivity of detectors for determination of $E$ and the fluctuation of the shower size. In the simulation, $\alpha^{\prime}$ is derived by convoluting the single power spectrum $E^{\alpha}$ with the energy distributions. And we determine the spectral index $\alpha$ by using the relation of $\alpha-\alpha^{\prime}$ shown in Figure 5

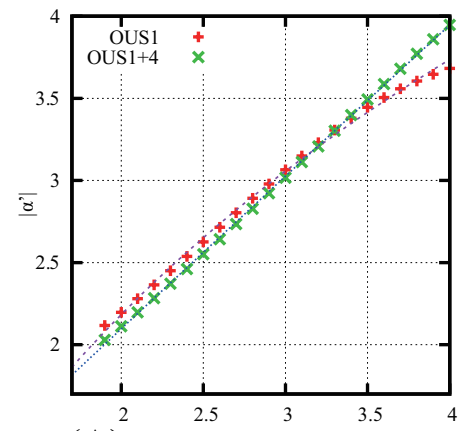

(A)

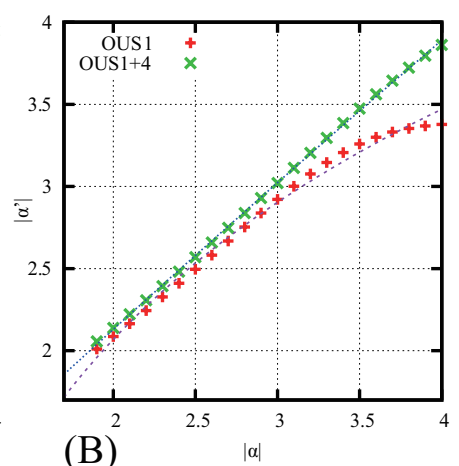

(B)

Figure 5: The comparison of proton primaries with irons in the relation between $\alpha$ and $\alpha^{\prime}$ obtained by the OUS1 (A) and the OUS1+4 (B). Dotted lines and dashed lines indicate results of best fitting.

In Figure 6 and Figure 7 we show the primary energy spectrum obtained from the OUS1 and the OUS1+4, respectively. The observed spectra are fitted by a single power law spectrum $E^{\alpha^{\prime}}$. The fitting is performed with the least square method and in the primary energy regions of (i) $10^{16} \mathrm{eV}$ to $10^{19.5} \mathrm{eV}$, (ii) $10^{16} \mathrm{eV}$ to $10^{18.5} \mathrm{eV}$ and (iii) $10^{18} \mathrm{eV}$ to $10^{19.5} \mathrm{eV}$, because the observed spectrum seems to be flattened around $E_{0}=10^{18} \mathrm{eV}$. In the OUS1, $\alpha^{\prime}$ (proton) and $\alpha^{\prime}$ (iron) are obtained as $-2.40 \pm 0.10$ and $-2.65 \pm 0.13$, respectively, in the primary energy range (i). By using the relation of $\alpha-\alpha^{\prime}$ shown in Figure 5 (proton) and $\alpha$ (iron) are equal to $-2.23 \pm 0.11$ and $-2.64 \pm 0.17$, respectively, in (i). In the primary energy region (ii), $\alpha^{\prime}$ (proton) and $\alpha^{\prime}$ (iron) are obtained as $-2.63 \pm 0.15$ and $-3.14 \pm 0.14$, respectively, in the OUS1. Thus, $\alpha$ (proton) and $\alpha$ (iron) are 
obtained as $-2.47 \pm 0.17$ and $-3.38 \pm 0.25$, respectively, in (ii).

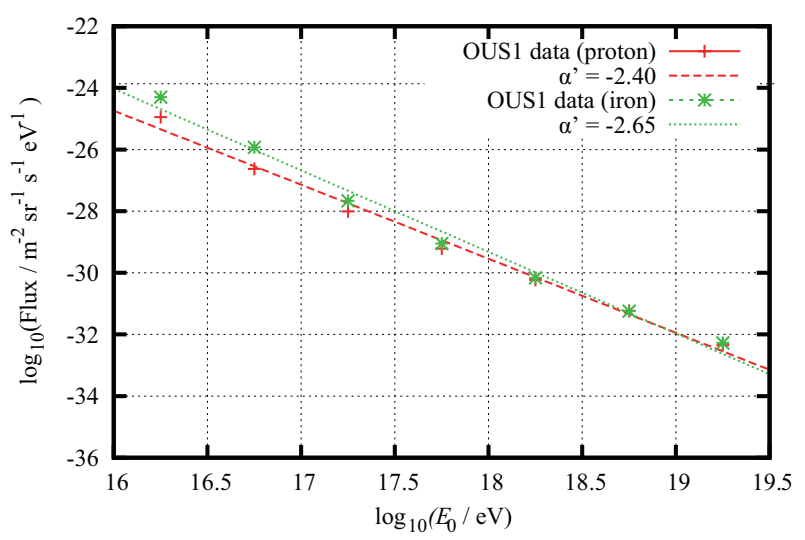

Figure 6: The observed spectrum assumed to be proton and iron primaries, respectively, in the OUS1. Experimental data are indicated by $(+)$ and $(*)$. The least square fitting are shown by lines.

We also determine the values of $\alpha$ in the OUS1+4 by using the same method as the OUS1. $\alpha$ (proton) and $\alpha$ (iron) are obtained as $-2.87 \pm 0.16$ and $-3.23 \pm 0.20$, respectively, in (i). In the primary energy region (ii), $\alpha$ (proton) and $\alpha$ (iron) are obtained as $-3.12 \pm 0.13$ and $-3.51 \pm 0.18$. The data spectral indies are summarized in the Table 2.

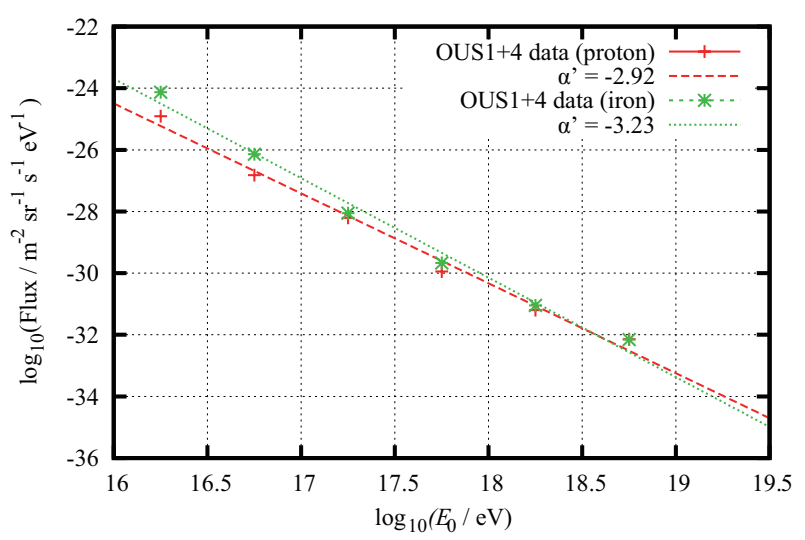

Figure 7: The observed spectra assumed to be proton and iron primaries, respectively, in the OUS1+4. Experimental data are indicated by $(+)$ and $(*)$. The least square fitting are shown by lines.

In this data analysis, the observed spectra assumed to be iron primaries show that their spectra are steeper than that assumed to be protons in both observations. The reason for this steepening effect is that the particle density of iron primaries at the ground level is lower than that of protons for the same primary energy, and data samples of which energies have been estimated to be $E_{0}<$ $10^{16} \mathrm{eV}$ in proton primaries are moved to higher energy region in irons.

\section{Conclusion}

The primary energy and its spectrum have been obtained by using compact EAS arrays and the 


\begin{tabular}{|c|c|c|c|c|}
\hline & \multicolumn{3}{|c|}{ Primary energy region $[\mathrm{eV}]$} \\
\hline & & (i) $10^{16}-10^{19.5}$ & (ii) $10^{16}-10^{18.5}$ & (iii) $10^{18}-10^{19.5}$ \\
\hline \multirow{2}{*}{$\begin{array}{l}\text { OUS1 } \\
\text { Proton }\end{array}$} & $\alpha^{\prime}$ & $-2.40 \pm 0.10$ & $-2.63 \pm 0.15$ & $-2.12 \pm 0.07$ \\
\hline & $\alpha$ & $-2.23 \pm 0.11$ & $-2.47 \pm 0.17$ & $-2.12 \pm 0.06$ \\
\hline \multirow{2}{*}{$\begin{array}{l}\text { OUS1 } \\
\text { Iron }\end{array}$} & $\alpha^{\prime}$ & $-2.65 \pm 0.13$ & $-3.14 \pm 0.26$ & $-2.11 \pm 0.03$ \\
\hline & $\alpha$ & $-2.64 \pm 0.17$ & $-3.38 \pm 0.25$ & $-2.04 \pm 0.03$ \\
\hline \multirow{2}{*}{$\begin{array}{l}\text { OUS1+4 } \\
\text { Proton }\end{array}$} & $\overline{\alpha^{\prime}}$ & $-2.92 \pm 0.15$ & $-3.14 \pm 0.25$ & - \\
\hline & $\alpha$ & $-2.87 \pm 0.16$ & $-3.12 \pm 0.13$ & - \\
\hline \multirow{2}{*}{$\begin{array}{l}\text { OUS1+4 } \\
\text { Iron }\end{array}$} & $\alpha^{\prime}$ & $-3.23 \pm 0.17$ & $-3.47 \pm 0.15$ & - \\
\hline & $\alpha$ & $-3.23 \pm 0.20$ & $-3.52 \pm 0.17$ & - \\
\hline
\end{tabular}

Table 2: The spectral index $\alpha^{\prime}$ and $\alpha$.

Linsley method, and by considering proton and iron primaries. In the comparison of the primary spectrum assumed to be proton primaries with that assumed to be irons, $\alpha$ (proton) and $\alpha$ (iron) are equal to $-2.47 \pm 0.17$ and $-3.38 \pm 0.25$, respectively, in the primary energy region of $10^{16} \mathrm{eV}$ to $10^{18.5} \mathrm{eV}$, in the OUS1. In the OUS4, $\alpha$ (proton) and $\alpha$ (iron) are obtained as $-3.12 \pm 0.13$ and $-3.47 \pm 0.17$, respectively, in the same primary energy region. Thus, taking the heavy compositions in the primary cosmic rays, the primary cosmic ray spectrum is suggested to be steeper than protons.

\section{References}

[1] A. Schulz et al., The measurement of the energy spectrum, in proceedings of 33rd ICRC Rio de Janeiro, (2013)

[2] D. Ikeda et. al., Ultra-high energy cosmic-ray, in proceedings of 32nd ICRC Bejing, (2011)

[3] M. I. Pravdin et. al., Search for photons, in proceedings of 31st ICRC Lodz, (2009)

[4] N. Ochi et al., Search for larg-scal coincidences, J. Phys. G: Nucl. Part. Phys., 2003, 29 1169-1180

[5] T. Wada et al., Observation of time correlation, Nucl. Phys. B (Proc. Suppl.), 1999, 75 (issue 1-2) $330-332$

[6] A. Iyono et al., Cosmic ray composition studies, Astrophysics and Space Sciences Transactions, 2011, $7327-333$

[7] J. Linsley, Thickness of the particle, J. Phys. G: Nucl. Phys., 198612 51-57

[8] M. Okita et al., Primary energy spectrum of, Nucl. Phys. B (Proc. Suppl.), 2008, 175-176, 322-325.

[9] H. Matsumoto et. al., Simulation studies and implementation, Nucl. Instr. \& Meth. A, 2010, 614 $475-482$

[10] S. J. Sciutto, AIRES, 1999, [astro-ph/9911331]

[11] S. Ostapchenko, Nonlinear screening effects, Phys. Rev. D, 2006, 74014026

[12] A. M. Hillas, Shower simulation, Nucl. Phys. B (Proc. Suppl.), 1997, 52(issue 3) 29-42 